\title{
Transfer of care - a randomised control trial investigating the effect of sending the details of patients' discharge medication to their community pharmacist on discharge from hospital \\ Megan Hocklya,b, Sian Williams ${ }^{\mathrm{b}}$ and Marcus Allen ${ }^{\mathrm{c}}$ \\ aPharmacy Department, Brighton and Sussex University Hospitals NHS Trust, bSchool of Pharmacy and Biomolecular Science, University of \\ Brighton, and 'Brighton and Sussex Centre for Medicines Optimisation, School of Pharmacy and Biomolecular Science, University of Brighton, \\ Brighton, UK
}

\begin{abstract}
Objective There is substantial evidence demonstrating that transferring patients between care providers is a high-risk area for medicines management. This study aimed to investigate the effect of sending patients' hospital discharge letters to their nominated community pharmacists on the number of discrepancies between the patient's general practitioner (GP) records and the discharge letter and between the patient's self-described medication regime and the discharge letter.

Methods In a randomised, controlled trial, 33 participants in two groups, control and intervention, had their discharge letter sent to either their GP only or their GP and nominated community pharmacy after hospital discharge. At least 3 weeks after hospital discharge, the participant's current GP's medication record and their self-described medication regime was obtained. Discrepancies between their GP medication record and their discharge letter and between the participant's self-described medication regime and their discharge letter were counted. The number of discrepancies (relative to the number of drugs prescribed) in the intervention group was compared with the control group for each of the above two categories, using the chi-squared test to determine the statistical significance of any differences between the two groups.

Results The intervention group had statistically fewer discrepancies than the control group for both data sets: GP records compared with the discharge letters $(P<0.0005)$; participants' self-described medication regimes compared with the discharge letters $(P<$ 0.00005).

Conclusions Sending a copy of patients' discharge letters to their community pharmacists could be beneficial in reducing postdischarge prescribing discrepancies and improving patient understanding of the changes made to their medicines.
\end{abstract}

Introduction

Transferring patients between care providers, such as on Within 2 weeks of hospital discharge, 57\% of patients hospital discharge, is a high-risk area for medicines man- experience medication problems and up to $20 \%$ experiagement. ${ }^{[1]}$ Better utilisation of community pharmacists ence adverse drug events (ADE). ${ }^{[2,3]}$ On hospital dis(CP) in the process of medicines management post-hospi- charge, $60 \%$ of patients have had three or more tal discharge may improve medicines reconciliation and medication changes and the risk of an ADE increases with patient safety. every new drug or alteration made. ${ }^{[4-6]}$ 
Communication of medication changes to the patient's general practitioner (GP) has improved immensely with the advent of electronic discharge letters. ${ }^{[7]}$ However, discrepancies and ADE post-discharge still continue to occur. The PRACtICE study ${ }^{[8]}$ found that $43 \%$ of patients had discrepancies between the medications prescribed on their discharge letter and those subsequently prescribed in practice.

Community pharmacists are ideally placed to help reduce drug-related problems. They are easily accessible, and $60 \%$ of patients use the same pharmacy regularly. ${ }^{[9]} \mathrm{A}$ previous study has shown CPs were able to identify drugrelated problems and intervene in 63\% of post-discharge patients. ${ }^{[6]}$ Many CPs now offer a wide range of services including Medicines Use Reviews (MUR). ${ }^{[10]}$ Although evidence is limited, post-discharge MURs are shown to have reduced hospital readmissions. ${ }^{[11]} \mathrm{CPs}$ are not currently sent any information on discharge medication and medication changes that have occurred. Sending CPs discharge letters allows them to check the discharge letter against the patient's next repeat prescription and identify any unintentional prescribing discrepancies. One study showed that when CPs are sent a discharge letter unintentional postdischarge prescription discrepancies fell from $57 \%$ to $32 \%{ }^{[12]}$ The number of ADE also fell from $3.1 \%$ to $1.6 \%$. In addition, a pilot study in Scotland demonstrated the benefits of sending discharge letters to CPs, and NHS Scotland has now instigated this system. ${ }^{[13]}$

Previous studies have focused on measuring post-discharge prescribing discrepancies and CP interventions for postdischarge patients..$^{[9,12,13]}$ However, one of the most important determinants in improving patient outcomes is how and if the patient takes their medicine. ${ }^{[14]}$ One study demonstrated that the majority of patients have little or no understanding of the medication changes made during their hospital admission. ${ }^{[15]}$ Patients' non-adherence to their medication leads to negative outcomes. Non-adherence results in $11-22 \%$ of hospitalisations for chronic disease exacerbations. ${ }^{[5]}$ It has been found that $4.3 \%$ of preventable hospital admissions are medication-related, ${ }^{[16]} 19 \%$ of hospital re-admissions are medication-related and $50 \%$ of the latter are preventable. ${ }^{[17]}$ It is therefore crucial to establish if changes made to a patient's medication regime whilst in hospital have been understood and followed by the patient as well as the other healthcare professionals involved in their care. Providing CPs with patients' discharge letters would allow them to check patients' understanding of any medication changes when they came to collect their repeat prescription. It would also alert the CP to offer the patient a post-discharge MUR.

This study aims to investigate the effect of sending a copy of patients' hospital discharge letters to their nominated CP as well as their GP surgery. It will measure the number of discrepancies between the hospital discharge letter and the patient's GP medication records

the patient's self-described medication regime

\section{Subjects and methods}

\section{Study design}

This randomised, controlled trial with two parallel arms $(1: 1)$ was conducted on 33 patients over 4 months at the Royal Sussex County Hospital (RSCH), Brighton, United Kingdom. Ethical approval was obtained from the South Central - Hampshire A National Research and Ethics Committee (reference number 13/SC/0513 on 7th Nov 2013), and informed written consent was obtained from all participants before enrolment.

\section{Participant recruitment}

Pharmacy staff opportunistically identified suitable participants for the researcher (not every patient could be assessed with available resources), from inpatients throughout RSCH. The researcher approached eligible patients and gave them verbal and written information on the study. After having at least 24 hours to consider, patients wishing to enrol gave written consent. Eligible participants needed to have taken at least four medicines regularly prior to hospital admission, used the same community pharmacy for all their repeat prescriptions, had changes made to their medication during their hospital stay and had their discharge letter clinically screened by a pharmacist. The agreement of their $\mathrm{CP}$ to participate in the trial was also required. CPs were faxed written information about the study, were asked for their agreement to participate and were requested to provide a named member of staff to link with the study. Patients with one or more of the following characteristics were excluded: under 18 years old; unable 
to carry out a telephone interview; with no registered GP; living outside Brighton and Hove; had their medication administered by carers; or dispensed in a blister pack.

Intervention

Once enrolled, participants were given a sequential study number which had previously been randomly allocated to either the control or intervention group using randomisation software to create a 'group allocation list'. Participants and the researcher were blinded to the group allocation, but hospital pharmacists who processed participants' discharge letters and the CPs were, of necessity, not blinded. The group allocation list and the group allocation of participants were kept from the researcher until the data had been processed.

When processing the discharge letter, hospital pharmacy staff used the group allocation list to determine the participant's study group. The control group followed standard discharge practice: the discharge letter was sent electronically to the GP only. The intervention group additionally had the discharge letter faxed to their nominated CP with a cover sheet explaining the trial. The CPs were not given instructions on what interventions they should make using the discharge letter but were told it could be used for post-discharge MURs. At least 3 weeks after discharge from hospital, each participant's current medication record was obtained from their GP surgery and a structured telephone interview with the participant was performed.

\section{Outcome measures and statistical methods}

The primary outcome measures of the study were:

Reconciliation, at least 3 weeks after discharge, between each participant's GP medication record and their hospital discharge medication, measured by the number of discrepancies between the participant's GP medication record and their hospital discharge letter.

Reconciliation, at least 3 weeks after discharge, between each participant's description of their amended medication regime and their hospital discharge medication, measured by the number of discrepancies between the participant's self-described medication regime and their hospital discharge letter.

For the purposes of this study, a discrepancy was classified as 'any difference in drug name, dose, route or frequency, between the two lists (the discharge letter and either the GP medication record or the participant's selfdescribed medication regime)'. Discrepancies in symptom relieving or short-term medicines such as analgesics, antibiotic courses and laxatives were not counted. The participant was asked whether they had had any changes made to their medications as discharge in the telephone interview to prevent intentional discrepancies from being counted. To reduce bias, all the analyses were carried out by a single pharmacist who was blinded to the participant's study group allocation. For each participant, discrepancies relative to the hospital discharge letter were counted for the GP medication records (GP data set) and for the participant's self-described medication regime (Pt data set). The total number of discrepancies for each data set was shown relative to the total number of drugs prescribed. The results for the control group and intervention group for each data set (GP and Pt) were compared on the number of discrepancies relative to the total number of drugs prescribed using the chi-squared test. A second analysis comparing the number of participants with one or more discrepancies was carried out also using the chi-square test. Both chi-square tests were one-tailed and tested for significance at $a=0.05$. The relative risk reduction (RRR) and number needed to treat (NNT) were calculated for the primary outcome measures. All statistical tests were performed with Minitab 17 and/or XLSTAT programmes. ${ }^{\text {[18,19] }}$

\section{Sample size}

The minimum required sample size was based on the first primary outcome measure and was estimated to be 32 using $G^{*}$ power 3 (calculated for a chi-squared test, $a=0.05, b=0.8$ and effect size ' $d$ ' $=0.5$ ). ${ }^{[20]}$ The effect size ' $d$ ' was estimated using data from a previous study. ${ }^{[12]}$ As the discrepancies are measured per number of drugs prescribed, the sample size relates to the number of drugs rather than the number of participants. Eligible patients must take at least four drugs and so a minimum of eight participants are required for confidence the sample size has been met. 


\section{Results}

\section{Study participant demographics and characteristics}

The study enrolled 33 participants over 4 months: 17 were randomised to the intervention group and 16 were randomised to the control group. Results were collected on all 33 participants for the GP data set (Figure 1) and 26 participants for the Pt data set (Figure 2). Results for seven participants in the Pt data set were lost to followup because they were un-contactable (4), had been readmitted to hospital at the point of data collection (2) or were too unwell to participate in the telephone interview (1).

The participant demographics, including the clinical speciality (respiratory, endocrine, etc.) under which the participant was admitted, are shown in Table 1. The two groups of the study, control and intervention, did not show any significant difference in comparison between the participants’ demographics (Table 1).

\section{Results for the primary outcome measures}

The 33 participants in the GP data set were prescribed a total of 364 drugs, and the 26 participants in the Pt data set were prescribed a total of 261 drugs at the point of discharge. The number of discrepancies relative to the number of drugs prescribed was significantly lower in the

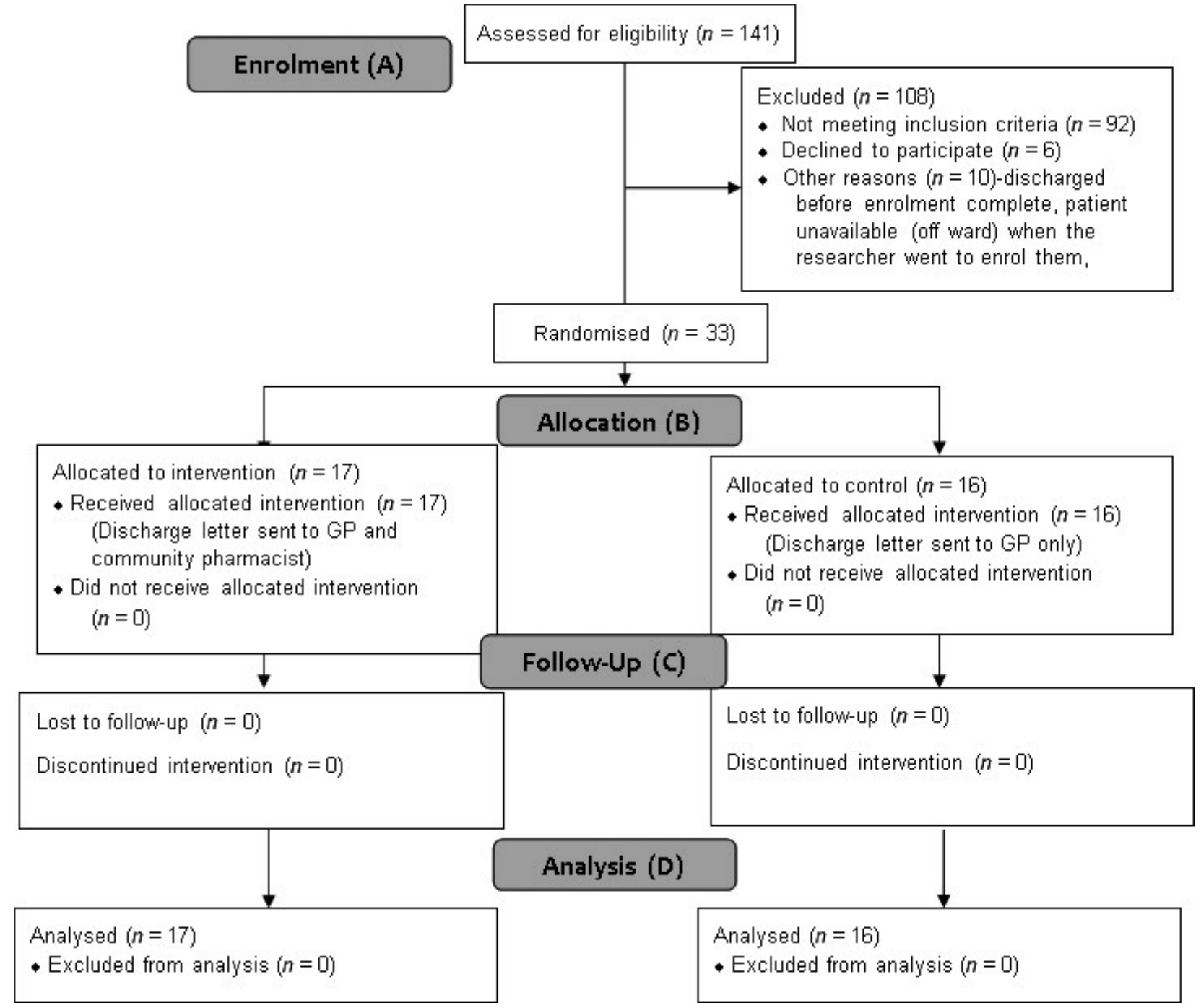


Figure 1 Consolidated Standards of Reporting Trials (CONSORT) diagram of the study process and participant flow for the set of results counting the discrepancies between the participants' GP medication records and their hospital discharge letters. A - Number who were assessed for eligibility and enrolled, with reasons given for those who were not enrolled, $\mathrm{B}$ - number who were allocated to each group control or intervention, $\mathrm{C}$ - number that were lost to follow-up and reasons why, D - number who were analysed. GP, general practitioner (see Appendix S1).

intervention group (I) than in the control (C) group in both the GP data set (I: $14 \%$, (25/173); C: $26 \%$, (50/191) P $=0.00034$ $(\mathrm{P}<0.05)$ ) and the Pt data set (I: 8\%, (10/128); C: 23\%, (31/133) $\mathrm{P}=0.000043(\mathrm{P}<0.05))$ (Table 2). The number of participants with at least one discrepancy was significantly lower in the intervention group (I) than in the control (C) group in both the GP data set (I: 27\%, (9/21); C: 36\%, (12/21) P = $0.029(\mathrm{P}<0.05))$ and the Pt data set (I: 19\%, (5/16); C: 42\%, (11/16) P = $0.0034(\mathrm{P}<0.05))$ (Table 2).

The GP data set showed a RRR of $29 \%$ (comparing the number of patients with at least one prescribing discrepancy) between the intervention and the control group and a NNT of four, meaning that for every four patients who have a copy of their discharge letter sent to their CP a prescribing discrepancy can be prevented. In the Pt data set, the RRR was 47\% with a NNT of two.

The mean number (standard error of the mean) of discrepancies observed per participant was lower in the intervention group than in the control group for both data sets, GP (I: 1.47 1.81; C: 2.94 3.34) and Pt (I: 0.83 1.11; C: 2.21 1.97) (Figure 3).

The number of major discrepancies observed was lower in the intervention group compared to the control in both the GP data set (I: 9; C: 19) and the Pt data set (I: 2; C: 3) (Table 3). 


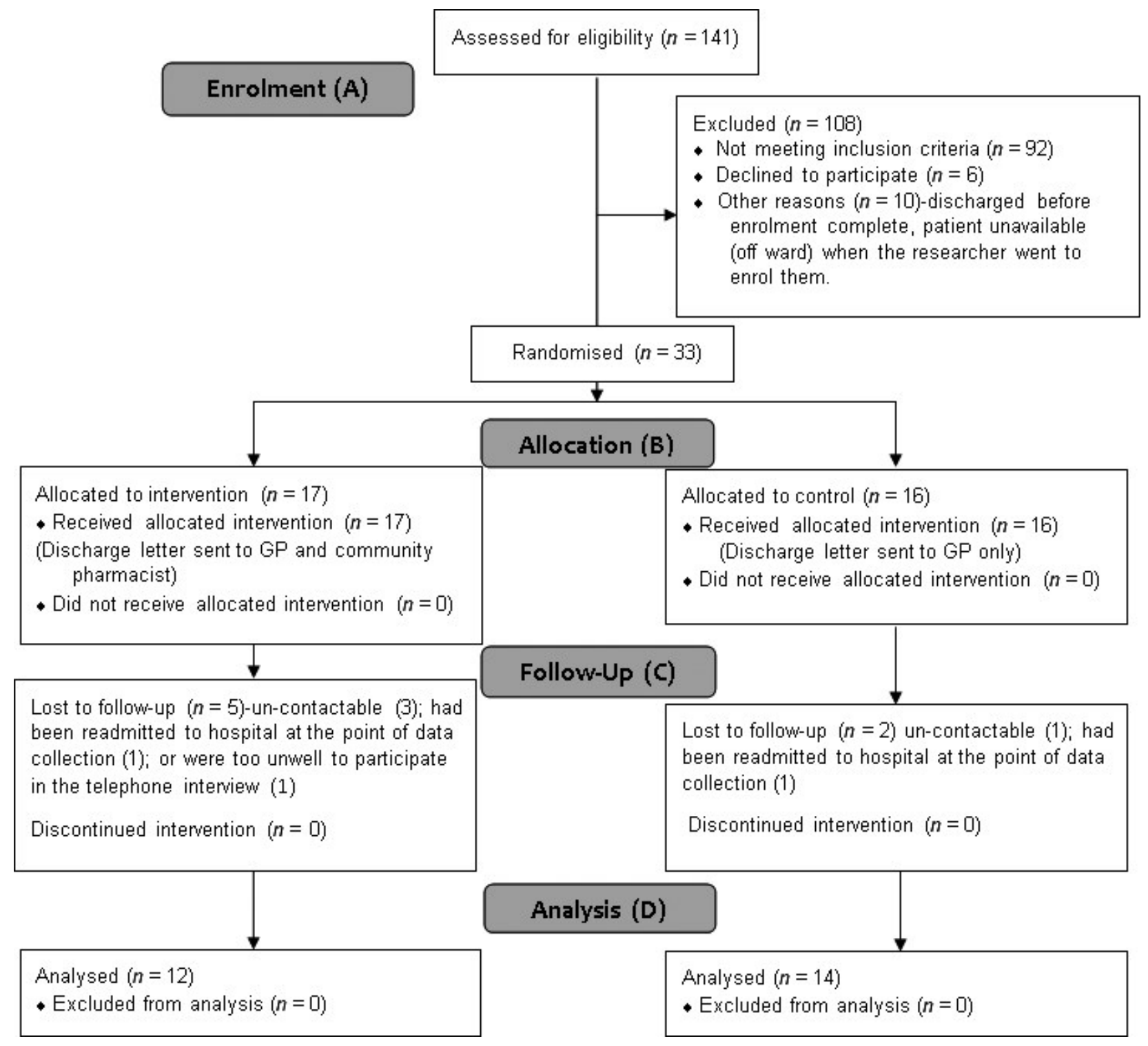

Figure 2 Consolidated Standards of Reporting Trials (CONSORT) diagram of the study process and participant flow for the set of results counting the discrepancies between the participants' self-described medication regimes and their hospital discharge letters. This differs from Figure 1 because some participants were lost to follow-up because they could not be contacted for the telephone interview (Section C). GP, general practitioner (see Appendix S1).

When counting discrepancies found in both the GP's medication records and the participants' self-described medication regime, that is a prescribing discrepancy that caused the patient to take the wrong medication/dose, the results are as follows:

- Control group: seven discrepancies (two major discrepancies)

- Intervention group: one discrepancy (minor)

None of the participants had received post-discharge MURs by the time the telephone interview was carried out. However, two participants had received counselling on their medication changes by their CP. Both of these participants were in the intervention group. 


\section{Discussion}

\section{Primary outcome measures}

The number of discrepancies relative to the number of drugs prescribed and the number of participants with at least one discrepancy was found to be significantly lower in the intervention group for the accuracy of both the GP

\begin{tabular}{lccc} 
Table 1 & Study population demographics, number of participants \\
\hline Study population demographic & Control & Intervention & P-value* \\
\hline Number of participants & 16 & 17 & \\
Male & 8 & 11 & 0.58 \\
Female & 8 & 6 & 0.52 \\
Mean age & 66 & 66 & 0.97 \\
$\begin{array}{l}\text { Mean number of medicines } \\
\text { Mean number of changes to }\end{array}$ & 11.94 & 10.18 & 0.39 \\
$\begin{array}{l}\text { medicines on hospital } \\
\text { discharge }\end{array}$ & 3.63 & 2.24 & 0.18 \\
\hline
\end{tabular}

Clinical Speciality

\begin{tabular}{lll}
\hline Respiratory & 7 & 7 \\
\hline Endocrine & 1 & 1 \\
\hline Care of the elderly & 1 & 0 \\
\hline Vascular & 1 & 4 \\
Medicine & 1 & 0 \\
\hline Gastro & 4 & 3 \\
\hline Cardiac & 1 & 0 \\
\hline Orthopaedics & 0 & 2 \\
\hline
\end{tabular}

Data presented as number of participants unless stated otherwise.

Taken as the number of medicines on the discharge letter.

* P-value results for the statistical tests performed to assess for differences in characteristics between the two study groups are given. The comparisons of the number of males and females between the control group and the intervention group were performed using the chisquared test; the comparisons of the mean age, mean number of medicines on the discharge letter and the mean number of changes to medicines at discharge between the control group and the intervention group were performed using the independent t-test. All tests were two-tailed and tested to the significance of a= 0.05 .

medication records and the participants' description of their amended medication regimes. The results therefore suggest that the sending of hospital discharge letters to

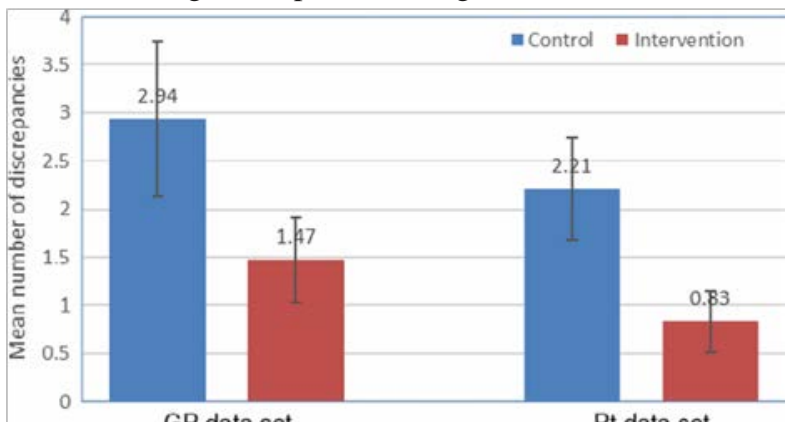

GP data set

Pt data set 
Figure 3 Mean number of discrepancies per participant in the control group and the intervention group when comparing the participants' GP records with their hospital discharge letters (GP data set) and the participants' self-described medication regimes with their hospital discharge letters (Pt data set). The error bars express the standard error of the mean discrepancies per participant. GP, general practitioner.

patients' CPs, a relatively simple and low cost intervention, is beneficial for both of the above measures. The incidence of medication discrepancies in the control group is relatively high for both measures (26\% GP data set and 23\% Pt data set), and so the scope for benefit of this intervention is significant.

The NNT is four for GP records and two for patients' self-described medication regimes. These low NNT values demonstrate the cost effectiveness of the intervention.

The study showed the intervention was able to reduce major discrepancies which can cause harm to the patient. Minor discrepancies should not to be overlooked though because whilst they may not directly lead Table 3 Number of major discrepancies in the control and intervention groups for both data sets (GP and Pt)

Table 2 The number and percentage of discrepancies in the control and intervention groups for both data sets, GP and Pt, and a comparison of the difference in discrepancies between the two groups

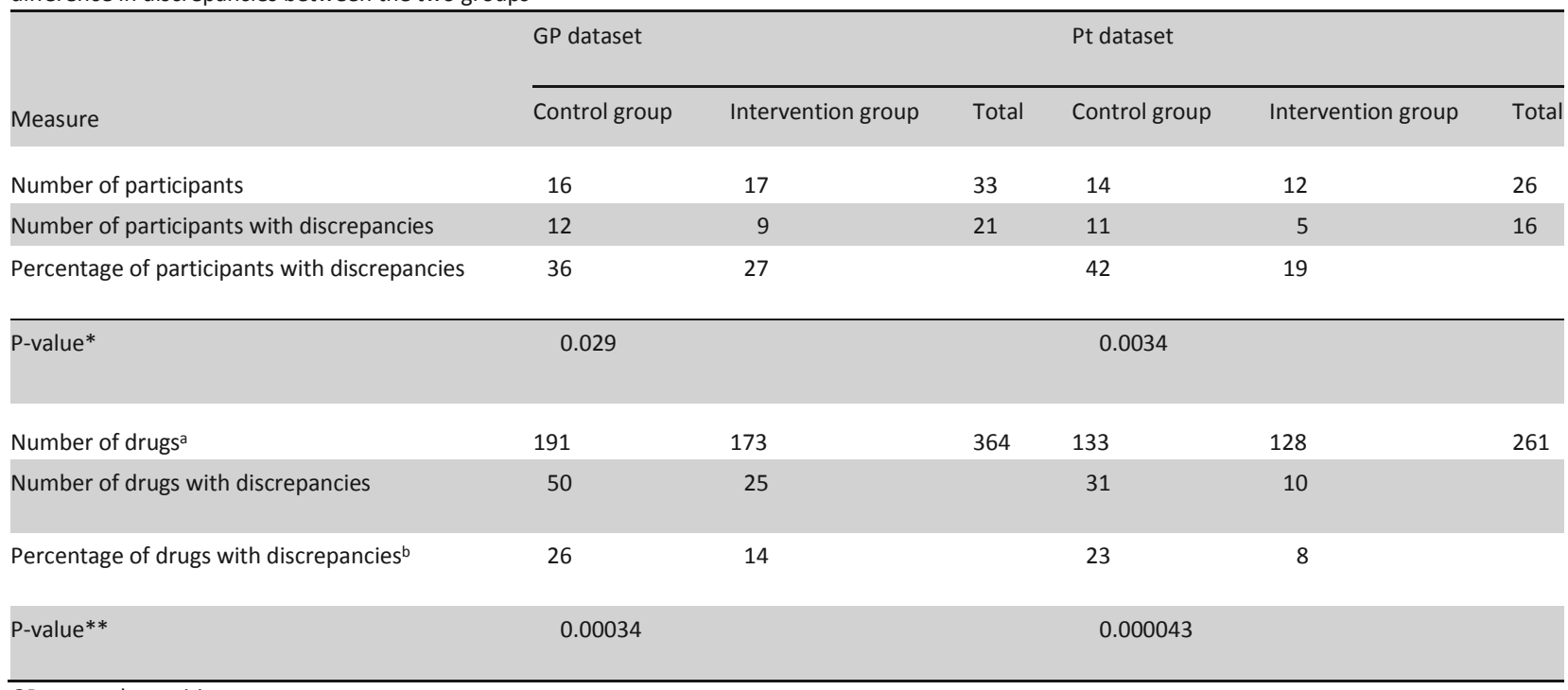

GP, general practitioner.

a

The number of drugs is taken as the number of drugs on the patient's discharge letter.

b Relative to the number of drugs.

*The chi-squared test was used to test for a statistical difference in the number of participants with at least one discrepancy between the two groups for each data set. A one-tailed test was used and tested to the significance of $a=0.05$.

**The chi-squared test was used to test for a statistical difference in the number of drugs with discrepancies between the two groups for each data set. A one-tailed test was used and tested to the significance of $a=0.05$.

\begin{tabular}{lll}
\hline & GP data set & Pt data set \\
& $\begin{array}{l}\text { Number of major } \\
\text { discrepancies }\end{array}$ & $\begin{array}{l}\text { Number of major } \\
\text { discrepancies }^{\mathrm{a}}\end{array}$ \\
\hline Control & 19 & 4 \\
Intervention & 9 & 2 \\
\hline
\end{tabular}

GP, general practitioner.

aFor the purposes of this study, a major discrepancy was classified as a discrepancy that could have caused an adverse effect on the patient. The results classed as major discrepancies were reviewed by a second pharmacist to reduce subjectivity. 
to harm, the long-term benefits of preventative therapy will be missed.

\section{Strengths and limitations}

Although the study showed a significant reduction in post-discharge medication discrepancies in patients who had their discharge letters sent to their CP compared to those who did not, the mechanism of the intervention is unclear.

The study postulated that CPs would use the discharge letter in two ways:

to identify patients suitable for post-discharge MURs, which would help improve patient understanding of their medicines (reduce discrepancies in participants' self-described medication regime)

to identify discrepancies on repeat prescriptions (reduce discrepancies in GP records).

Although CPs were made aware of the objectives of the study (with references to MURs and to handling of repeat prescriptions), the CPs were not given any specific instructions as to what interventions they should make in response to receipt of the discharge letter.

Investigation and analysis of the CPs' interventions were beyond the resources of this study and so it is unclear what interventions may have been carried out by the CPs prior to the data collection activities, to help bring about the measured reductions in discrepancies.

None of the participants reported having had a postdischarge MUR. Factors which may have prevented a post-discharge MUR happening are that not all community pharmacies in the area can carry out MURs, and that many patients get their medicines delivered, which decreases contact with their CP and reduces the opportunity for initiation of a MUR. MUR guidance states that they should ideally be conducted within 4 weeks of discharge, but for reasons of practicality up to 8 weeks is allowed. ${ }^{[10]}$ As the average time from discharge to the telephone interview was 26 days, this study may have timed the data collection too early to detect MURs which did take place, but at a later time.

Impact of GP record discrepancies on the participants' medication regimes

The intervention was found to work well because it helped to reduce discrepancies in both GP records and patient medication regimes. Ensuring that the patient takes the right medicine at the right time is a key objective in medicines optimisation. ${ }^{[21]}$

General practitioner record discrepancies do not always correlate with discrepancies in the patient's self-described medication regime as shown in this study. There was a much higher incidence of major discrepancies in the GP data set than the Pt data set and of the discrepancies found, few occurred in both the GP's medication records and the participant's selfdescribed medication regimes. This suggests the intervention impacts the discrepancies in the participant's self-described medication regime by a

mechanism other than by reducing GP prescribing discrepancies.

The lack of correlation between GP discrepancies and participant discrepancies could be for a number of reasons: because some of the participants had not yet collected repeat prescriptions and were still using medicines issued from the hospital; because the participant understood the medication changes and so ensured they took the correct medication despite discrepancies on the GP records; or because there are many additional factors that can affect patients' adherence with their medication regimes.

\section{Issues relating to GP medication records}

A key issue found when assessing prescribing discrepancies was inconsistency in GP surgery medication record systems and processes. Different surgeries have different computer systems and processes for updating records after receiving a discharge letter. For example, the updating of medication records may be performed by clinical or by non-clinical staff. Interventions aimed at reducing post-discharge GP prescribing discrepancies would need to overcome these issues or a more standardised practice in updating GP records should be sought.

\section{Directions for future research}

The need to understand CPs' interventions in response to the discharge letter has been highlighted as a key area for further investigation by this study. A future study in which CPs are asked to record the number and type of interventions they make 
using discharge letters would further advance this line of research. CPs could be asked to record intervention types through categorisation similar to that used by Paulino et al. ${ }^{[9]}$ Ideally this would be conducted on a much larger scale than the present study, recruiting around 150-200 patients and engaging all CPs in the area. The study should have a longer follow-up period, for example 8-10 weeks, to see if this led to the detection of more MURs. Data on discrepancies between the patients' GP records and their hospital letter, and the patients' self-described medication regime and their hospital letter should be collected in conjunction with the data on the CPs' interventions. This would allow analysis of the effect the CPs' interventions had on the number and type of discrepancies detected.

\section{Conclusion}

The present work shows that sending a copy of each patient's discharge letter to their nominated CP could be beneficial in reducing post-discharge prescribing discrepancies and improving patient understanding of the changes made to their medicines. To implement this change in procedure, a convenient method of transfer of information, ideally electronic, would need to be found. Difficulties in liaising with community pharmacies outside the local area served by a hospital would have to be overcome. To ensure the intervention is cost-effective and benefits those most in need, a system for identifying and targeting high-risk patients for post-discharge medication discrepancies should be employed.

\section{Declarations}

\section{Conflict of interest}

The Author(s) declare(s) that they have no conflicts of interest to disclose.

\section{Funding}

This research received no specific grant from any funding agency in the public, commercial or not-for-profit sectors.

\section{Acknowledgements}

This work has been read and approved by all the above authors, all authors had access to the study data, and the authors meet the requirements for authorship as defined by the International Committee of Medical Journal Editors.

\section{Authors' contributions}

All authors contributed to the conception and design of the work, the acquisition, analysis and interpretation of data for the work, drafting and revising the work and gave final approval of the version to be published and accountable for all aspects of the work.

\section{Ethical approval}

South Central - Hampshire A National Research and Ethics Committee, reference number 13/SC/0513 on 7th Nov 2013.

\section{References}

1. Picton C, Wright H (2012). Keepingpatients safe when they transfer between care providers - getting the medicines right [report on the internet]. London: Royal Pharmaceutical Society. http://www.rpharms.com/cur rentcampaigns-pdfs/rps-transfer-of-ca refinal-report.pdf (Archived by WebCite at http://www.webcitation. org/6ha8Nxh1Q) (accessed 12 July 2014).
2. Sexton J, Brown A. Problems withmedicines following hospital discharge: not always the patient's fault? J Soc Adm Pharm 1999; 16: 199-207.

3. Gray SL, Mahoney JE, Blough DK.Adverse events in elderly patients receiving home health services following hospital discharge. Ann Pharmacother 1999; 33: 1147-1153.

4. Himmel $\mathrm{W}$ et al. Drug changes at the interface between primary and secondary care. Int J Clin Pharmacol Ther 2004; 42: 103-109.
5. Thompson-Moore

$\mathrm{N}$,

Liebl MG.

Health care systems vulnerabilities: understanding the root causes of patient harm. Am J Health Syst Pharm 2012; 69: 431-436.

6. Paulino EI et al. Drug related problems identified by European pharmacists in patients discharged from hospital. Pharm World Sci 2004; 26: 353-360.

7. Motamedi SM et al. The efficacy of computer-enabled discharge communication interventions: 
systematic review. BMJ Qual Saf 2011; 20: 403-415.

8. Avery T et al. (2012). An Investigation into the prevalence and cause of prescribing errors in general practice: The PRACtICE study, a report for the GMC [report on the internet]. London: The General Medical Council. http://www.gmc-uk.org/Investigating_ the_prevalence_and_causes_of_prescri bing_errors_in_general_practice_ The_PRACtICe_study_Reoprt_May_ 2012_48605085.pdf. (Archived by WebCite at http://www.webcita tion.org/6ha8d57E5) (accessed 18 December 2012).

9. Central Office of Information. Community Pharmacy Use Quantitative and Qualitative Research: Market Research Report. London: Department of Health, 2008.

10. Pharmaceutical Services Negotiating Committee (2014). Advanced services [website on the internet]. London: Pharmaceutical Services Negotiating Committee. http://psnc.org.uk/service scommissioning/advanced-services/.

(Archived by WebCite at http:// www.webcitation.org/6ha8lR5K9) (accessed 23 July 2014).

11. Colquhorn A. MURs help free hospital beds. Pharma J 2010; 285: 615.

12. Duggan C. Reducing adverse prescribing discrepancies following hospital discharge. Int J Pharm Pract 1998; 6: 77-82.

13. Burns H et al. (2013). Safer use of medicines: medicines reconciliation: revised definition, goals and measures and recommended practice statements for the Scottish safety programme. [report on the internet]. Edinburgh: The Scottish Government. http://www. sehd.scot.nhs.uk/cmo/CMO(2013)18. pdf (accessed 24 July 2014).

14. Boswell KA et al. Associating medication adherence with improved outcomes: a systematic literature review. Am J Pharm Benefits 2012; 4: e97-e108. [serial on the internet].

15. Ziaeian B et al. Medicines accuracy and patient understanding of intended medicine changes on hospital discharge. J Gen Intern Med 2012; 27: 1513-1520.

16. Winterstein AG et al. Preventable drugrelated hospital admissions. Ann Pharmacother 2002; 36: 1238-1248.

17. Barry A (2013). A study of medications related readmissions in patients discharged within one month from the medical admissions units [conference presentation on the internet]. Edinburgh: Society for Acute Medicine. http://www.acutemedicine.org.uk/ wpcontent/uploads/2013/05/barry.pdf

(Archived by WebCite at http:// www.webcitation.org/6haAqMzgF) (accessed 23 July 2012).

18. Minitab 17 Statistical Software [Computer software]. State College, PA: Minitab Inc, 2010

19. XLSTAT. MS Excel [Computer software]. New York: Addinsoft, 2007

20. Faul F et al. G*Power 3 [Computer software]. Dusseldorf: Heinrich-Heine University, 2007.

21. Picton C, Wright H (2013). Medicines optimisation: Helping patients make the most of their medicines. [report on the internet]. London: Royal Pharmaceutical Society. http:// www.rpharms.com/promoting-phar macy-pdfs/helping-patients-makethemost-of-their-medicines.pdf (accessed 3 November 2014).

\section{Supporting information}

Additional Supporting Information may be found in the online version of this article at the publisher's website:

Appendix S1. CONSORT 2010 checklist of information to include when reporting a randomised trial. 